\title{
Cytoskeletal Breakdown and Apoptosis Elicited by NO Donors in Cerebellar Granule Cells Require NMDA Receptor Activation
}

\author{
*Emanuela Bonfoco, †Marcel Leist, *Boris Zhivotovsky, *Sten Orrenius, \\ $\ddagger$ Stuart A. Lipton, and $* \nmid$ Pierluigi Nicotera

\begin{abstract}
*Institute of Environmental Medicine, Karolinska Institutet, Stockholm, Sweden; $\uparrow$ Faculty of Biology, University of Konstanz, Konstanz, Germany; and \$Laboratory of Cellular and Molecular Neuroscience, Children's Hospital, and Program in Neuroscience, Harvard Medical School, Boston, Massachusetts, U.S.A.
\end{abstract}

\begin{abstract}
We have recently demonstrated that nitric oxide (NO) donors can trigger either apoptosis or necrosis of neurons as a function of the intensity of the exposure. Here, we show that the apoptosis induced by the NO donors S-nitrosocysteine (SNOC) or S-nitroso- $N$-acetylpenicillamine (SNAP) in cultured cerebellar granule cells (CGCs) depends on NMDA receptor (NMDA-R) activation leading to intracellular $\mathrm{Ca}^{2+}$ overioad. Early dissolution of actin filaments followed by breakdown of microtubules and nuclear lamins preceded the appearance of typical apoptotic features. NO donors induced tyrosine nitration in neurons, in a small population of contaminating astrocytes, and in cultures of cerebellar astroglial cells. However, astrocytes neither displayed cytoskeletal alterations nor underwent apoptosis. Competitive and uncompetitive NMDA receptor antagonists, such as Daminophosphonovaleric acid and MK-801, did not influence tyrosine nitration but prevented the accumulation of intracellular $\mathrm{Ca}^{2+}$, cytoskeletal breakdown, and apoptosis induced by either SNOC or SNAP in CGCs. Taken together, these data strongly suggest that $\mathrm{Ca}^{2 .}$ influx through NMDA-R-gated ion channels is a critical event in CGC apoptosis induced by NO donors. Key Words: Apoptosis - Calcium-Cytoskeleton-Neurodegeneration-Nitric oxide.

J. Neurochem. 67, 2484-2493 (1996).
\end{abstract}

Nitric oxide (NO) plays a major physiological role in the nervous system (Garthwaite and Boulton, 1995). However, inappropriate generation of this mediator-possibly in conjunction with reactive oxygen species - has been also implicated in neuropathology (Zhang and Snyder, 1995). Neurotoxicity of NO and related species has been attributed, at least in part, to DNA damage followed by activation of poly (ADPribose) synthase, with consequent $\mathrm{NAD}^{+}$and ATP depletion (Zhang et al., 1994). However, NO toxicity may also originate from direct protein modifications. The NO group, in its various redox forms, can inhibit iron sulfur enzymes and some nonheme iron proteins and thereby contribute to a breakdown in cellular energy supplies and ribonucleotide synthesis (Gross and Wolin, 1995). Furthermore, peroxynitrite $\left(\mathrm{ONOO}^{-}\right)$ generated from $\mathrm{NO}^{*}$ and $\mathrm{O}_{2}{ }^{\circ}$ ' (Stamler et al., 1992) can react with tyrosine residues, causing alterations of protein phosphorylation or perturbation of protein tertiary structure (Beckman et al., 1994). Finally, protein thiol groups are targets of NO-related species, and $S$-nitrosylation modifies protein function (Lipton et al., 1993). The individual importance of damage to such targets may be dependent on the mode of exposure and on the cell type.

The understanding of NO pathophysiology is also complicated by its interaction with other second messengers and mediators. Especially important in neurons is the interaction between $\mathrm{NO}$ and glutamate signaling systems. On one hand, NO can induce the release of neurotransmitter molecules (Hanbauer et al., 1992), such as glutamate (O'Dell et al., 1991; Montague et al., 1994). On the other hand, glutamate-stimulated $\mathrm{Ca}^{2+}$ signals elicit NO production by a $\mathrm{Ca}^{2+}$ - and calmodulin-sensitive NO synthase (Garthwaite et al., 1988; Bredt and Snyder, 1989). Such endogenously produced NO mediates, at least in part, glutamate toxicity in rat cortical cultures (Dawson et al., 1991). The

Resubmitted manuscript received May 30, 1996; accepted July 12, 1996.

Address correspondence and reprint requests to Prof. P. Nicotera at Faculty of Biology, University of Konstanz, Box 5560-X911, D78434 Konstanz, Germany

Abbreviations used: Aiv, acetoxymethyl ester; APV, D-aminophosphonovaleric acid; BAPTA, 1,2-bis (2-aminophenoxy)ethane$N, N, N^{\prime}, N^{\prime}$-tetraacetic acid; $\left[\mathrm{Ca}^{2+}\right]$, intracellular $\mathrm{Ca}^{2+1}$ concentration; CGC, cercbellar granule cell; FITC, fluorescein isothiocyanate; GFAP, glial fibrillary acidic protein; NMDA-R, NMDA receptor; $\mathrm{NO}$, nitric oxide; $\mathrm{ONOO}^{-}$, peroxynitrite; PBS, phosphate-buffered saline; SNAP, $S$-nitroso- $N$-acetylpenicillamine; SNOC, $S$-nitrosocysteine; SOD, superoxide dismutase; TRITC, tetramethylrhodamine $B$ isothiocyanate. 
redox species of NO is also important in determining the biological effect. For example, $\mathrm{NO}^{*}$ reacts with $\mathrm{O}_{2}{ }^{-\cdots}$ to produce $\mathrm{ONOO}^{-}$and its associated neurotoxicity, whereas $\mathrm{NO}^{+}$decreases NMDA-receptor (NMDAR) channel activation, thereby attenuating $\mathrm{Ca}^{2+}$ overload elicited by glutamate (Lipton et al., 1993); vice versa, there may be NO redox species that increase the intracellular $\mathrm{Ca}^{2++}$ concentration $\left(\left[\mathrm{Ca}^{2+}\right]_{i}\right.$ ) (Richter et al., 1994).

In our previous work, we have demonstrated that cortical neurons or cerebellar granule cells (CGCs) undergo apoptosis after mild insult delivered by NO donors or NMDA-R agonists (Ankarcrona et al., 1995; Bonfoco et al., 1995a). Here, we examine whether $\mathrm{Ca}^{2+}$ signals are involved in the apoptosis elicited by NO donors. We investigated the effect of agents that either lowered $\left[\mathrm{Ca}^{2+}\right]_{i}$ or prevented $\mathrm{Ca}^{2+}$ entry through NMDA-R channels after exposure to NO donors. Furthermore, because cytoskeletal damage can promote apoptosis of CGCs (Bonfoco et al., 1995b), we decided to investigate whether cytoskeletal alterations were also part of NO-induced neuronal apoptosis and the possible role of $\mathrm{Ca}^{2+}$ in their development.

\section{MATERIALS AND METHODS}

\section{Chemicals}

$N$-Lauroylsarcosine, poly-L-lysine ( $\mathrm{mol} \mathrm{wt}, 300,000$ ), Lcysteine, Hoechst 33258, phalloidin-fluorescein isothiocyanate (FITC), and mouse monocional antibodies against $\alpha$ tubulin, $\beta$-tubulin, or glial fibrillary acidic protein (GFAP) were obtained from Sigma. Phalloidin-tetramethylrhodamine B isothiocyanate (TRITC), propidium iodide, SYTO-13, and the acetoxymethyl ester's (AMs) of 1,2-bis (2-aminophenoxy) ethane- $N, N, N^{\prime}, N^{\prime}$-tetracetic acid (BAPTA), calcein, and fura 2 were purchased from Molecular Probes (Eugene, OR, U.S.A.). MK-801 and D-aminophosphonovaleric acid (APV) were from Research Biochemicals International (Natick, MA, U.S.A.). S-Nitroso-N-acetylpenicillamine (SNAP) was purchased from Calbiochem (San Diego, CA, U.S.A.). S-Nitrosocysteine (SNOC) was prepared as described previously (Lei et al., 1992).

\section{Cell cultures and exposure}

CGCs were prepared from 7-day-old Sprague-Dawley rats, as described by Schousboe et al. (1989a) with small modifications (Ankarcrona et al., 1995). Glial cells were prepared as described (Hansson and Rönnbäck, 1989) and cultured in Eagle's basal medium supplemented with $10 \%$ heat-inactivated fetal calf serum. Culture purity $(>95 \%)$ was assessed in each preparation using GFAP staining as a marker for glial cells.

NO donors were added from stock solutions directly to the culture medium. In the experiments examining the effect of cell density on cytotoxicity, CGCs were seeded in wells containing a coverslip. Before the exposure, coverslips were removed to leave in the weils a small but intact ring of CGCs. Alternatively, after replacing the culture medium with an analogous, serum-free medium, a fraction of cells was gently scraped off, the dishes were washed free of possible debris, and the remaining intact adherent patch of cells was reincubated in the original culture medium. NMDA-R antagonists ( $1 \mu M \mathrm{MK}-801$ or $10 \mu M \mathrm{APV}$ ) or superoxide dismutase (SOD; $5 \mathrm{kU} / \mathrm{ml}$ ) was added as $200 \times$ concentrated stock solutions $10 \mathrm{~min}$ before the treatment with the NO donors.

\section{Tyrosine nitration}

After exposure, CGCs adherent to glass coverslips were fixed with $80 \%$ (vol/vol) methanol for $15 \mathrm{~min}$ at room temperature, rinsed in phosphate-buffered saline (PBS), and treated with $3 \%$ (vol/vol) $\mathrm{H}_{2} \mathrm{O}_{2}$ for $5 \mathrm{~min}$ to remove endogenous peroxidase activity. Mouse monoclonal IgG ( $1: 60$ dilution) or rabbit polyclonal (1:80) antibodies directed against nitrotyrosine (a kind gift of Dr. J. S. Beckman, University of Birmingham, Birmingham, AL, U.S.A.) were then added to the coverslips, which were incubated for $1 \mathrm{~h}$ at $25^{\circ} \mathrm{C}$ followed by incubation with a biotinylated secondary antibody. A DAKO LSAB kit based on the peroxidase reaction was used as the detection system (Beckman et al., 1994). A positive reaction yields a red precipitate at the antigen site. Mayer's hematoxylin solution was used for counter staining.

\section{Microscopy}

Celis were visualized either using a conventional fluorescence microscope (Leica, DM-IRB) or a scanning confocal microscope (Bio-Rad MRC 600 or Leica TCS-4D). The TCS-4D microscope was equipped with a high-power argon laser (UV), a krypton/argon laser (visible), and three independent fluorescence detectors. Confocal images were calibrated digitally using as reference an object micrometer. The horizontal width of the white figure labels serves as the calibration bar.

\section{$\left[\mathrm{Ca}^{2+}\right]_{\mathrm{i}}$ measurements}

Fluorescence measurements of $\left[\mathrm{Ca}^{2+}\right]_{i}$ in fura 2-loaded CGCs or glial cells were made using a SPEX Fluorolog-2 system as described previously (Bonfoco et al., 1995b). Changes in the ratio of fiuorescence intensities, with excitation wavelengths of 340 and $380 \mathrm{~nm}$, were measured at predetermined times after exposure to NO donors or other additions. The emission was set at $510 \mathrm{~nm}$, and $\left[\mathrm{Ca}^{2+4}\right]_{i}$ was calculated according to the method of Grynkiewicz et al. (1985).

\section{Formation of apoptotic nuclei}

Apoptotic nuclei were detected by propidium iodide staining (Bonfoco et al., 1995b) and scored in 15-20 fields for each slide. Their number was expressed as a percentage of total neuronal cells visible in the fields. We also doublestained glial and neuronal cultures with $10 \mu M$ ethidium bromide and $600 \mathrm{n} M$ calcein-AM to visualize cells with intact plasma membranes (calcein positive) versus cclls with broken membranes (ethidium bromide positive).

\section{Analysis of DNA fragmentation on agarose gels}

Formation of high-molecular-weight DNA fragments was detected by field inversion gel electrophoresis as described previously in detail (Ankarcrona et al., 1995). Calibration of DNA sizes was performed using sets of pulse markers: chromosomes from Saccharomyces cerevisiae $(225-2,200$ $\mathrm{kb})$ and $\lambda$-DNA ( $48.5 \mathrm{kbp})$. Gels were stained with ethidium bromide, visualized using a UV light source $(305 \mathrm{~nm})$, and photographed with Polaroid 665 positive/negative film. Oligonucleosomal DNA fragmentation was detected after DNA extraction from lysates of CGCs $\left(1 \times 10^{7}\right)$, as described elsewhere (Ankarcrona et al., 1995). Agarose gel electro- 
phoresis was performed with a constant voltage $(90 \mathrm{~V})$ for $3 \mathrm{~h}$ (Wyllie, 1980).

\section{Immunocytochemistry for cytoskeletal proteins}

After exposure, coverslips were rinsed in PBS and fixed (2.5\% paraformaldehyde for actin, a sequence of $80 \%$ methanol and acetone for $\beta$-tubulin, and a sequence of $2.5 \%$ paraformaldehyde and $0.2 \%$ Triton X-100 for $\alpha$-tubulin and GFAP). For $\beta$-tubulin, coverslips were incubated at $37^{\circ} \mathrm{C}$ for $30 \mathrm{~min}$ with a mouse monoclonal antibody used at a 1:200 dilution. Then coverslips were rinsed in PBS and incubated with a secondary antibody [anti-mouse $\operatorname{IgG~} \mathrm{F}(\mathrm{ab})_{2}$ fragment, fluorescein-conjugated] for $30 \mathrm{~min}$ at $37^{\circ} \mathrm{C}$. For F-actin staining, coverslips were incubated for $20 \mathrm{~min}$ at room temperature with $100 \mathrm{n} M$ phalloidin conjugated with either FITC or Texas Red. Coverslips werc then rinsed with PBS and mounted in HEPES-buffered saline/glycerol (1:1 $\mathrm{vol} / \mathrm{vol}$ ) on microscope slides. Lamin staining was performed after permeabilization with $100 \%$ methanol for 15 min using an antibody kindly provided by Dr. John Eriksson (Åbo Akademi, Finland). Blocking of nonspecific staining was done with PBS and $1 \%$ bovine serum albumin for 15 min before incubation with rabbit anti-lamin antibodies (1:60) for $30 \mathrm{~min}$ at room temperature. Coverslips were rinsed three times with PBS and then exposed for 30 min to secondary antibodies (Texas Red-conjugated goat anti-rabbit, 1:80 dilution). After rinsing with PBS, coverslips were exposed to the nuclear stain SYTO- $13(2 \mu M)$ for 20 min at room temperature. Subsequently, they were rinsed with PBS and mounted on glass slides for microscopy.

\section{Determination of glutamate concentrations}

Glutamate concentrations in cell culture supernatants were measured using an enzymatic assay kit (Boehringer, Mannheim, Germany). The assay is based on the formation of NADH catalyzed by glutamate dehydrogenase. The NADH subsequently reduces a tetrazolium dye in a diaphorase-mediated reaction. The detection limit was $2 \mu M$ glutamate, as determined experimentally.

\section{RESULTS}

\section{NO donors induce apoptotic cell death in CGCs}

SNOC $(200 \mu M)$ or SNAP $(200 \mu M)$ triggered CGC death with characteristic apoptotic features. Following exposure to either of the two NO donors, the number of cells displaying condensed, highly fluorescent nuclei increased significantly between 6 and $18 \mathrm{~h}$ and finally reached $\sim 75 \%$ after 24 h (Fig. 1). Results with SNOC or SNAP were identical, whereas SNOC preincubated for $24 \mathrm{~h}$ to release NO before exposure had no effect. For convenience, only the results with SNOC are illustrated. To score the number of viable cells remaining on the dishes after treatment, we stained cultures with the combination of calcein-AM plus ethidium bromide. Typically, membrane permeability of CGCs treated with SNOC or SNAP increased at late time points. Nuclei of apoptotic cells appeared condensed and red compared with viable green cells after $18 \mathrm{~h}$ (Fig. 2a
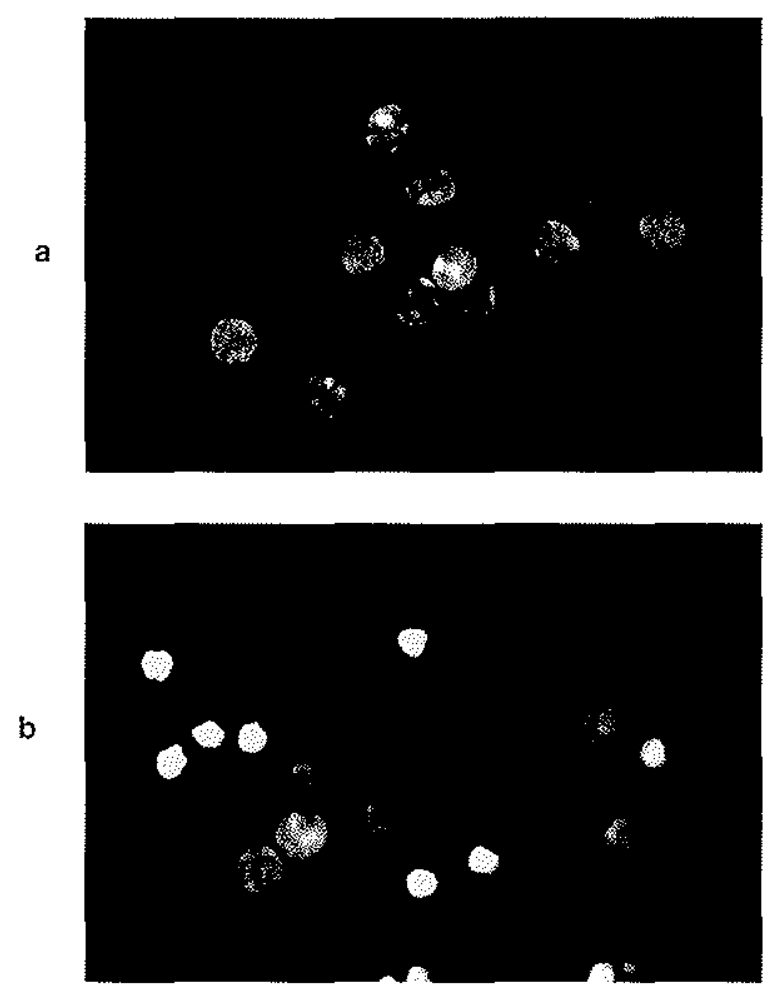

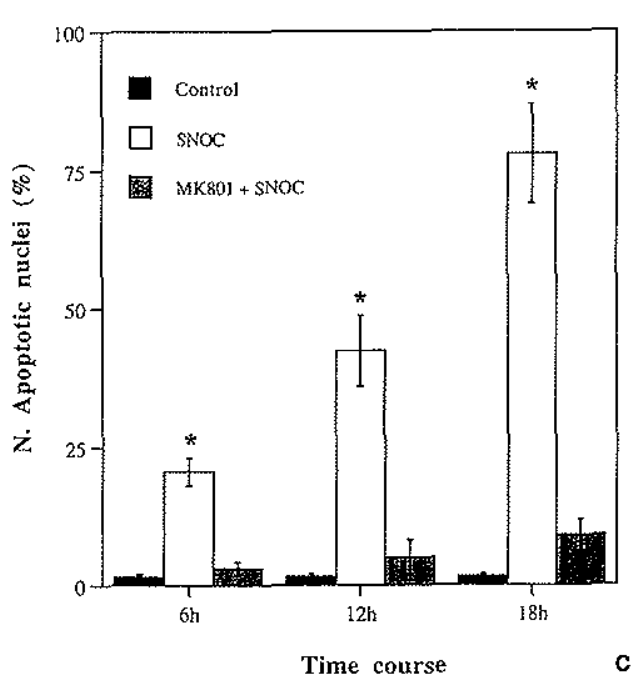

c

FIG. 1. Time course of formation of apoptotic nuclei in CGCs after exposure to $200 \mu M$ SNOC. Cells in (a) untreated cultures and (b) cultures after a 12-h exposure were stained with propidium lodide; (c) apoptotic nuclei were counted in 10-15 fields in each culture and normalized to the total number of nuclei. MK801 + SNOC refers to preincubation with $1 \mu M$ MK-801 for 5 min before exposure to SNOC. Apoptotic bodies were counted at the time points indicated. ${ }^{\star} p<0.01$ as determined by Student's $t$ test. 

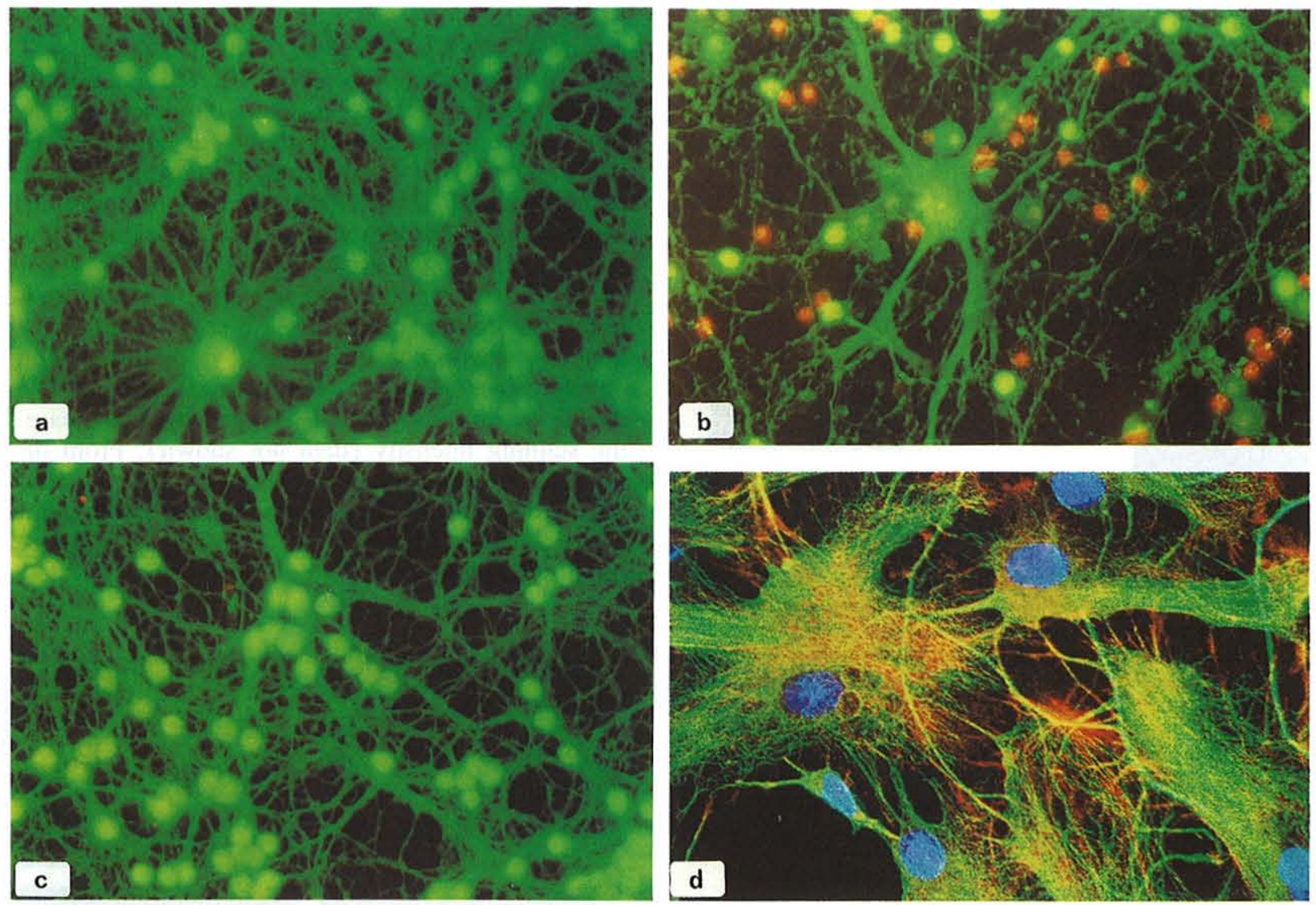

FIG. 2. SNOC induces death in neurons but not in astrocytes. CGC cultures were stained with calcein-AM plus ethidium bromide 18

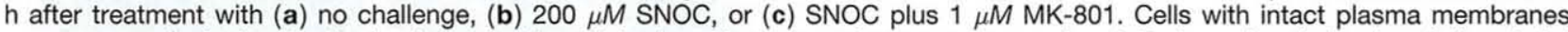
appear green (calcein retention), whereas cells with broken plasma membranes appear red and display pyknotic (and partially zeiotic) nuclei. Note in (b) that the single astrocyte present in the field was not affected by SNOC; (d) glial cultures were exposed to SNOC for $18 \mathrm{~h}$ and subsequently fixed. A triple stain was obtained using $1 \mu \mathrm{g} / \mathrm{ml}$ Hoechst 33258 (blue nuclei), rhodamine-coupled phalloidin (red actin filaments), and anti- $\alpha$-tubulin-FITC-conjugated monoclonal antibodies (green microtubule structures). Images were collected using a Leica TCS-4D confocal microscope. The white label serves as the calibration bar, which corresponds to a length of $18 \mu \mathrm{m}$ for $(\mathrm{a}-\mathrm{c})$ and $11 \mu \mathrm{m}$ for $(\mathrm{d})$.

and b). In contrast, the few GFAP-positive cells present in our cultures, as well as pure astrocyte cultures treated with NO donors, showed neither necrosis nor apoptosis up to 5 days after the treatments (Fig. $2 b$ and d). To establish whether endogenous NO generation would contribute to the NO donor toxicity, we preincubated cultures with $\mathrm{N}$-monomethylarginine before the exposure to SNOC or SNAP. We found no difference in the extent of apoptosis in the cells treated with the inhibitor of NO synthase, in agreement with other findings in similar models (Demerle-Pallardy et al., 1991).

$\mathrm{ONOO}^{-}$formed from $\mathrm{NO}^{-}$and $\mathrm{O}_{2}{ }^{-}$has been implicated as a major neurotoxic species. Here, the involvement of $\mathrm{ONOO}^{-}$is suggested by the observation that pretreatment of CGCs with SOD reduced SNOC-induced apoptosis: After $18 \mathrm{~h}$, untreated CGC cultures contained $7 \pm 2 \%$ apoptotic neurons, whereas treatment with SNOC caused apoptosis in $77 \pm 10 \%$ of the neuronal population, and cultures pretreated with SOD had only $40 \pm 6 \%$ apoptotic cells.

\section{DNA fragmentation in CGCs exposed to NO donors}

Progressive DNA cleavage to high- and low-molecular-weight fragments became evident $3 \mathrm{~h}$ after addition of NO donors. At the time the first apoptotic bodies were observed (6h), 50-kbp fragments were also visible on gels. The results obtained with SNOC are illustrated in Fig. 3. Oligonucleosomal-sized $(<20$ $\mathrm{kbp)}$ DNA fragments were detected by conventional agarose gel electrophoresis beginning $12 \mathrm{~h}$ after exposure to NO donors (Fig. 3b).

\section{Cytoskeletal alterations in CGC apoptosis induced by NO donors}

Because CGCs exposed to cytoskeletal poisons undergo apoptosis with a time course similar to that observed in this study (Bonfoco et al., 1995b), we decided to investigate whether early cytoskeletal alterations were also a feature of the apoptotic program induced by the NO donors. We examined the early effects of SNAP or SNOC on actin filaments, microtu- 
a

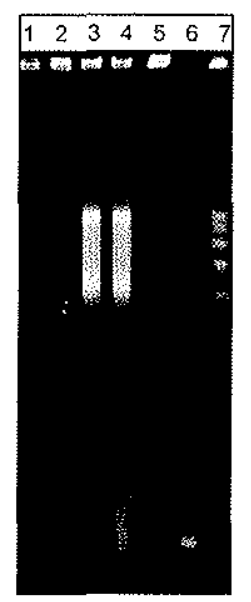

FIG. 3. Progressive formation of high-molecular-weight DNA fragments and DNA laddering in CGCs exposed to $200 \mu \mathrm{M}$ SNOC. Neurons were scraped off the plates and processed for DNA analysis by field-inversion gel electrophoresis (FIGE) and conventional agarose gel electrophoresis. a: FIGE gel. Lane 1 control; lane 2, $3 \mathrm{~h}$ after SNOC exposure; lane 3, $6 \mathrm{~h}$ after SNOC; lane 4,12 h after SNOC; lane 5, $12 \mathrm{~h}$ after SNOC in CGCs pretreated with $1 \mu M$ MK -801 ; lane 6, DNA molecular weight marker, $48 \mathrm{kbp}$; and lane 7, DNA molecular weight markers, 200-700 kbp. b: Conventional agarose gel electrophoresis. Lane 1, control; lane 2, $3 \mathrm{~h}$ after SNOC treatment; lane 3, after $12 \mathrm{~h}$; lane 4 , after $24 \mathrm{~h}$.

bules, and lamins. Figure 4 illustrates the effect of SNOC on F-actin. As early as $1 \mathrm{~h}$ after exposure, actin filaments showed a lack of continuity (Fig. 4b). At 3 $\mathrm{h}$ after SNOC treatment, F-actin was clearly fragmented, and by $12 \mathrm{~h}$ the marked loss of actin fluorescence was indicative of a generalized depolymerization (Fig. 4c and d). F-actin dissolution was followed by microtubule fragmentation, which became conspicuous $6 \mathrm{~h}$ after addition of SNOC (Fig. $4 \mathrm{f}$ and g). Nuclear lamins also disintegrated after exposure to SNOC, coinciding with the formation of high-molecular-weight DNA fragments and the appearance of apoptotic bodies (Fig. 5). Phase-contrast microscopy revealed that the axonal structure was still intact when the microtubules and actin filaments had already fragmented. Thus, cytoskeletal changes involving actin filaments clearly preceded the appearance of distinct nuclear changes. Astrocytes did not display alterations in GFAP, microtubule, or actin filament staining (Figs. $2 \mathrm{~d}$ and $4 \mathrm{~d}$ ).

\section{Exposure to NO donors results in tyrosine} nitration in both CGCs and astroglial cells

The experiments with SOD suggested that formation of $\mathrm{ONOO}^{-}$was at least in part involved in the apoptosis elicited by NO donors. Nitration of tyrosines has been implicated in toxicity and cytoskeletal alteration induced by $\mathrm{ONOO}^{-}$(Beckman et al., 1994).
Because apoptosis and cytoskeletal damage occurred extensively in CGCs treated with NO donors, we examined whether this would be reflected in a concomitant nitration of tyrosine residues. To detect nitrotyrosine in situ, we used either mono- or polyclonal antibodies in parallel incubations. Fifteen minutes after addition of SNAP or SNOC to CGC cultures, both neurons and astrocytes were positively stained regardless of the antibody used (Fig. 6a, b, and f). Staining persisted with similar intensity also in cells exposed to the NO donors for $>1 \mathrm{~h}$. As a negative control, the antibody reaction was quenched by addition of $1 \mathrm{~m} M$ nitrotyrosine (Fig. 6e). Pretreatment with SOD reduced tyrosine nitration, as judged by a decrease in the staining intensity (data not shown). From these findings, we conclude that although tyrosine nitration occurred in both neurons and astrocytes, additional factors influenced the initiation of apoptosis in the neuronal population.
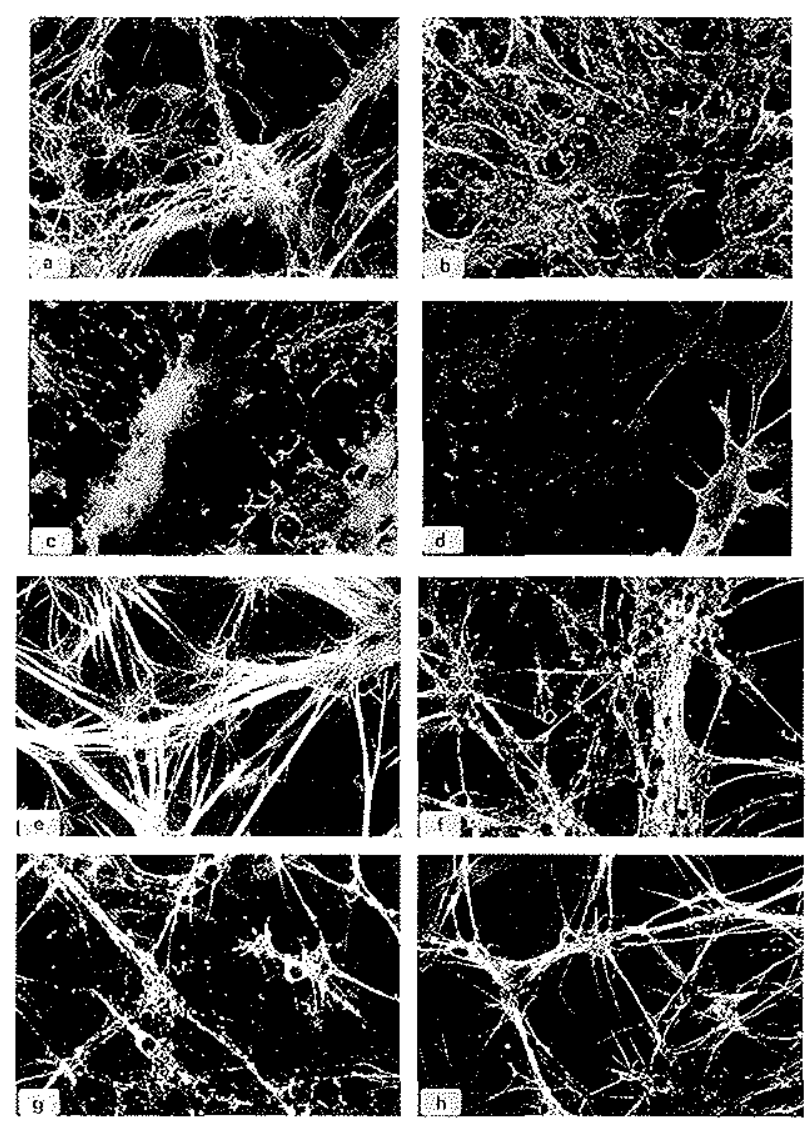

FIG. 4. F-actin and $\beta$-tubulin in CGCs exposed to $200 \mu M$ SNOC. F-actin immunostaining was performed in neuronal cultures: control (a) and 1 (b), 6 (c), and $12 \mathrm{~h}$ (d) after SNOC treatment. Note in (d) the intact actin structure in the glial cell present in the field. $\beta$-Tubulin immunostaining was also done in control cultures (e) and 6 ( $f$ ) and $12 \mathrm{~h}(\mathrm{~g}$ ) after SNOC treatment; (h) $\beta$-tubulin $12 \mathrm{~h}$ after treatment with $1 \mu \mathrm{M} \mathrm{MK}-801$ and 200 $\mu M$ SNOC. The white label that serves as the calibration bar corresponds to a length of $16(a-d)$ or $20 \mu \mathrm{m}(e-h)$ in the original sample. 

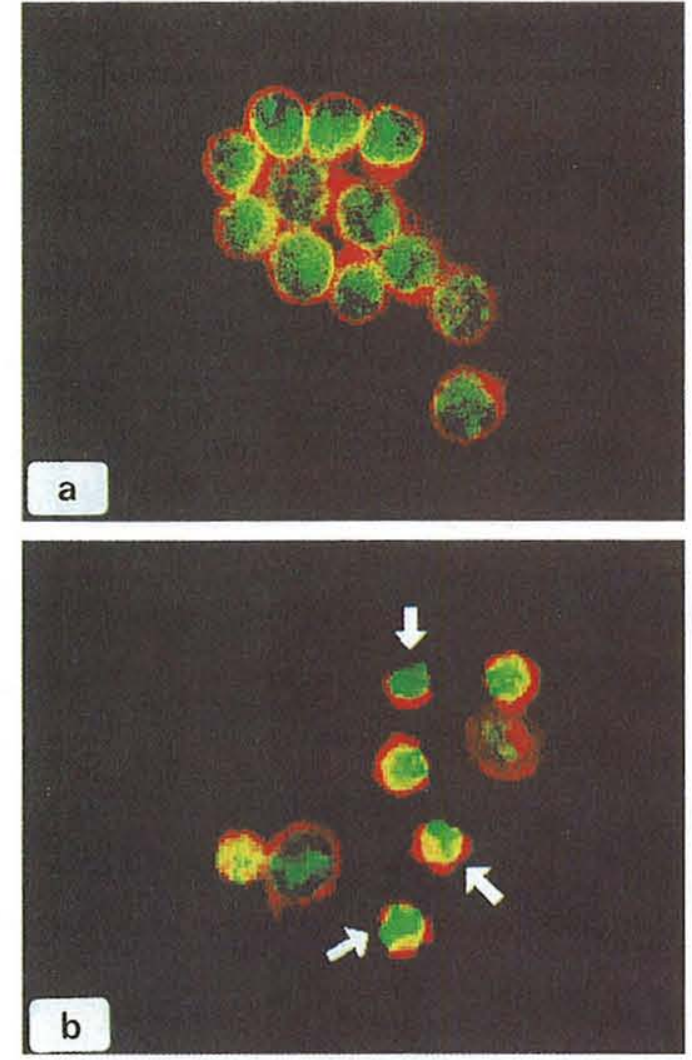

FIG. 5. Immunostaining of lamins in CGCs after NO treatment: (a) control and (b) $12 \mathrm{~h}$ after of $200 \mu \mathrm{M}$ SNOC exposure. Lamins are stained red (TRITC-coupled secondary antibody). Nuclear counterstaining was performed using the green fluorescent dye SYTO-13. Arrows indicate lamin breakdown in condensed nuclei. The white label that serves as the calibration bar corresponds to a length of $10 \mu \mathrm{m}$ in the original sample.

\section{Treatment with SNOC increases $\left[\mathrm{Ca}^{2+}\right]_{i}$ and} alters $\mathrm{Ca}^{2+}$ responses to high $\mathrm{K}^{+}$levels

We decided to investigate whether other signals commonly eliciting neuronal apoptosis, i.e., $\mathrm{Ca}^{2+}$ overload, could be involved in this system. We initially measured $\left[\mathrm{Ca}^{2+}\right]_{\mathrm{i}}$ in individual neurons using fura 2AM. Within $30 \mathrm{~min}$ of the addition of either of the two NO donors, the resting $\left[\mathrm{Ca}^{2+}\right]_{\mathrm{i}}$ increased from a control value of $\sim 40 \mathrm{n} M$ to an average of $90 \mathrm{n} M$. After $3 \mathrm{~h}$ in treated $\mathrm{CGCs},\left[\mathrm{Ca}^{2+}\right]_{\mathrm{i}}$ had increased further to reach a resting level of $120 \mathrm{n} M$ (Fig. 7a). Treatment with the NO donors also caused alterations in stimulated $\mathrm{Ca}^{2+}$ responses. Exposure of untreated CGCs to high $\mathrm{K}^{+}$levels caused a rapid and pronounced increase in $\left[\mathrm{Ca}^{2+}\right]_{i}$, whereas in neurons previously exposed to NO donors for $3 \mathrm{~h}$, the same stimulus did not increase $\left[\mathrm{Ca}^{2+}\right]_{i}$ further (Fig. 7). In contrast, resting $\left[\mathrm{Ca}^{2+}\right]_{i}$ in glial cells $(40 \pm 6 \mathrm{n} M)$ was unchanged after treatment with NO donors. To determine whether $\mathrm{Ca}^{2+}$ entry through neuronal membrane channels was involved, we selected either of two NMDA-R antagonists, MK-801 or APV. Either of these agents entirely prevented the increase in $\left[\mathrm{Ca}^{2+}\right]_{\mathrm{i}}$ induced by either
SNOC or SNAP and also restored the response to stimulation with high $\mathrm{K}^{+}$levels (Fig. $7 \mathrm{~b}$ and $\mathrm{c}$ ).

\section{Prevention of cytoskeletal alterations and apoptotic cell death by MK-801, an NMDA-R or open-channel blocker}

Next, we investigated whether restoring the resting $\left[\mathrm{Ca}^{2+}\right]_{\mathrm{i}}$ would also delay or prevent the toxicity of the NO donors. When CGCs were pretreated with MK801 , apoptosis was prevented even at late time points (Fig. 2). In addition, cytoskeletal structures remained intact (Fig. 4h), and DNA fragmentation as well as the late loss of membrane permeability did not occur (Figs. $3 \mathrm{a}$ and 1c). Because $\mathrm{Mg}^{2+}$ is known to inhibit NMDA-R activation, we tested whether raising $\mathrm{Mg}^{2+}$ concentrations in the culture medium would also protect from apoptosis induced by the NO donors. Indeed, increasing the $\mathrm{Mg}^{2+}$ concentration in the medium to 5 or $10 \mathrm{mM}$ reduced the formation of apoptotic nuclei elicited by SNOC; $18 \mathrm{~h}$ after SNOC treatment there were $77 \pm 10 \%$ apoptotic nuclei, whereas in the presence of $10 \mathrm{mM} \mathrm{Mg}{ }^{2+}$, the number of cells with apoptotic features decreased to $25 \pm 5 \%$. Furthermore, when the intracellular $\mathrm{Ca}^{2+}$ buffering capacity was temporarily increased by loading cells with $10 \mu M$ BAPTA-AM before exposure to SNOC or SNAP, apoptotic cell death was also attenuated $(8 \mathrm{~h}$ after SNOC treatment there were $31 \pm 4 \%$ apoptotic nuclei in the absence and $15 \pm 3 \%$ apoptotic nuclei in the presence of intracellular BAPTA).

NMDA-R activation can lead to the production of $\mathrm{O}_{2}{ }^{-}$in cultures of CGCs or cortical neurons (LafonCazal et al., 1993; Dugan et al., 1995). Thus, NMDA$\mathrm{R}$ activation may have mediated the formation of $\mathrm{ONOO}^{-}$and the consequent downstream events, i.e., tyrosine nitration. To investigate this possibility, we determined tyrosine nitration in CGCs treated with the NO donors in the presence of NMDA-R antagonists (either MK-801 or APV). As shown in Fig. 6c and d, these NMDA antagonists did not noticeably affect the nitration of tyrosine residues induced by SNOC.

\section{Role of endogenous NMDA-R agonists in CGC apoptosis induced by NO donors}

Finally, we investigated whether an endogenously released mediator or a component from the cell culture medium, such as glutamate, was responsible for the activation of NMDA-R channels in CGCs treated with NO donors. The mean glutamate concentration in the medium of CGCs exposed to NO donors remained consistently below $2 \mu M$, i.e., a concentration that does not affect the viability of CGCs if added directly to the cell culture medium. Thus, the enhanced glutamate release, if any, that occurred during incubation with the NO donors was not sufficient to increase the overall glutamate concentration in the medium. Pretreatment of neurons with $1 \mathrm{~m} M$ pyruvate plus $35 \mathrm{U} / \mathrm{ml}$ glutamate pyruvate transaminase to degrade glutamate did not protect neurons from toxicity induced by NO donors. Finally, to examine further whether an increase 

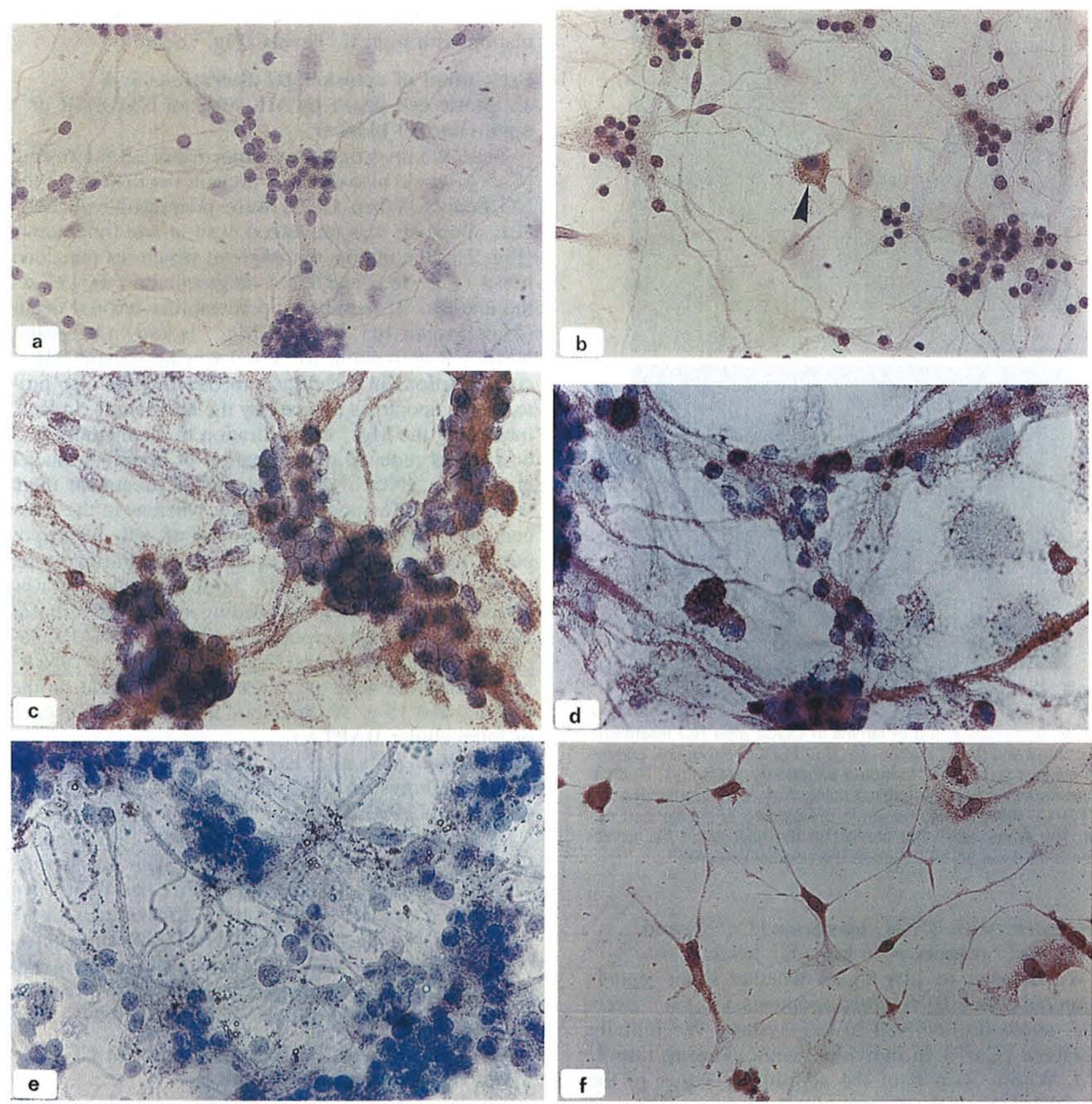

FIG. 6. Immunochemical detection of nitrotyrosine formation after treatment with SNOC. Cultures were fixed and stained with mono-

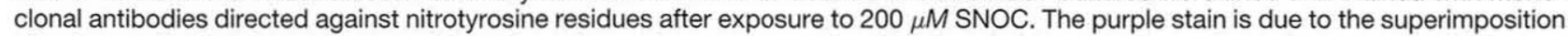
of red, specific for nitrotyrosine, and blue from the nuclear counterstain with Mayer's hematoxylin. a: Control neurons. b: CGCs and a single astrocyte (arrowhead) exposed to SNOC. c: Cultures pretreated with $1 \mu M$ MK-801 and then exposed to SNOC as in (b). d: As in (c) except that pretreatment was with $10 \mu \mathrm{M}$ APV. e: Negative control shows competitive blocking of primary antibody with 1 $\mathrm{mM}$ free nitrotyrosine. $\mathrm{f}$ : Cerebellar astrocytes treated with SNOC. The white label used as the calibration bar corresponds to 23 (a, $\mathrm{b}$, and f) or $15 \mu \mathrm{m}(\mathrm{c}-\mathrm{e})$.

in the overall concentration of any NMDA-R agonist in the culture medium was involved, we designed experiments in which neuronal cell densities were reduced to $\sim 10 \%$ (see Materials and Methods for experimental details). We found that neither the time course and relative extent of apoptosis nor the sensitivity to MK-801 changed under these conditions.
Nevertheless, a local release of glutamate (or other NMDA-R agonists such as aspartate) may have produced a stimulation at protected synaptic sites. To test this hypothesis, we investigated whether APV, a glutamate antagonist that prevents the activation of the NMDA-R by inhibiting agonist binding, would block the toxic effects of SNOC or SNAP in CGCs. Indeed, 
FIG. 7. $\left[\mathrm{Ca}^{2+}\right]$ in $\mathrm{CGCs}$ after treatment with SNOC. a: CGCs exposed to $200 \mu M$ SNOC were loaded with $2 \mu M$ fura 2-AM 30 min before the time point indicated. Following depolarization with $50 \mathrm{mM} \mathrm{KCl}$ $\left[\mathrm{Ca}^{2+}\right]_{1}$ concentrations were recorded. The mean $\mathrm{Ca}^{2+}$ concentrations for four to six different neurons are displayed. Pretreatment of CGCs with (b) $1 \mu \mathrm{M}$ MK-801 or (c) $10 \mu M$ APV (AP5) prevented the increase in resting $\left[\mathrm{Ca}^{2+}\right]_{i}$ and restored a normal re sponse to $\mathrm{KCl}$ depolarization $2 \mathrm{~h}$ after exposure to $200 \mu M$ SNOC.

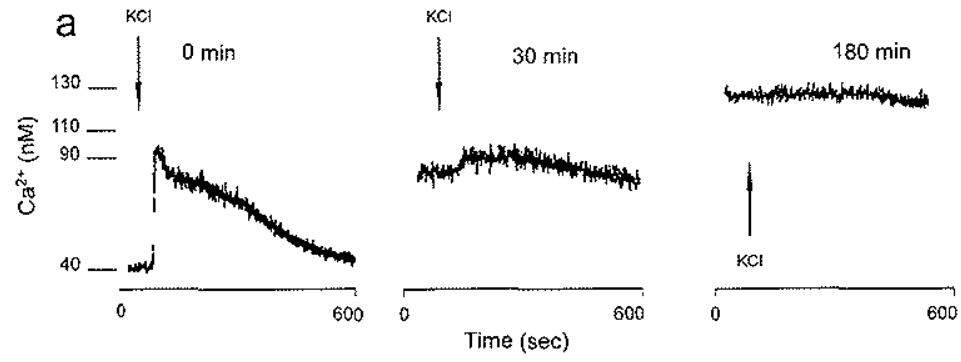

b

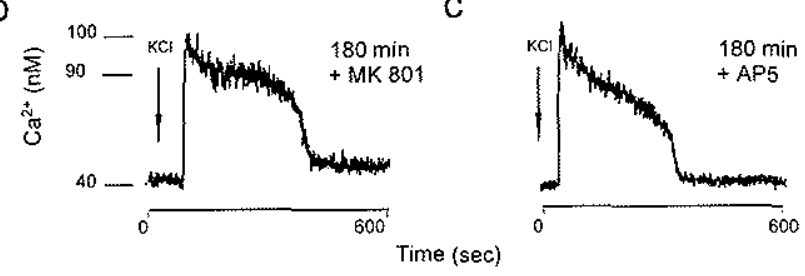

pretreatment with APV prevented the increase in restm ing $\left[\mathrm{Ca}^{2+}\right]_{i}$ and restored the response to high $\mathrm{K}^{+}$levels compromised by treatment with the NO donors (Fig. $7 \mathrm{~b}$ and $\mathrm{c}$ ). Also, cytoskeletal alterations, DNA fragmentation, and apoptosis elicited by SNOC or SNAP were prevented. Moreover, MK-801 or APV protected neurons from apoptosis when added up to $1 \mathrm{~h}$ after SNOC or SNAP ( 71 and $83 \%$ of the neuronal population were rescued by $M K-801$ and APV, respectively, as determined $18 \mathrm{~h}$ after SNOC treatment). Altogether, these results are consistent with the notion that the ONOO " (possibly together with other NO-related species) triggers the release of a glutamate-like agonist close to NMDA-R sites.

\section{DISCUSSION}

In our previous work, we have shown that mild insults with $\mathrm{NO}^{\circ}$ in conjunction with $\mathrm{O}_{2}{ }^{-\cdots}$ or NMDA-R agonists elicit apoptosis in cerebrocortical cultures and CGCs (Ankarcrona et al,, 1995; Bonfoco et al. $1995 a$ ). Here, we went on to examine possible mechanisms by which NO donors can trigger neuronal apoptosis in the CGC model system. The advantage of these cultures is the homogeneity of the neuronal population, the low content of nonneuronal cells, and the absence of confounding factors such as the dependence of neuronal survival on growth factors and accessory cells.

Our results show that apoptosis elicited by NO donors in CGC cultures requires a $\mathrm{Ca}^{2+}$ signal triggered by activation of NMDA-R channels. The most likely explanation for these findings is that NO or a downstream species, e.g., $\mathrm{ONOO}^{-}$formed in the presence of $\mathrm{O}_{2}{ }^{* \cdots}$, stimulates the release of NMDA-R agonists, which subsequently act on NMDA-Rs. This is apparent from the findings that mechanistically different inhibitors of NMDA-Rs, MK-801 (Wong et al., 1986) and APV (Davies et al., 1981), prevented the increase in the resting $\left[\mathrm{Ca}^{2+}\right]_{i}$ and the onset of cytoskeletal alterations and apoptosis elicited by the NO donors. Further evidence for this supposition comes from the neuroprotective effects of $\mathrm{Mg}^{2+}$ in this culture system. Potential endogenous agonists include the classical amino acid neurotransmitters glutamate and aspartate (Watkins and Evans, 1981), which are both released from CGCs on depolarization (Levi et al., 1984; Schousboe et al., $1989 \mathrm{~b}$ ) or other low-molecular-weight substances, such as quinolinic acid. NO can stimulate neurotransmitter release (Hanbauer et al., 1992; Montague et al., 1994), and it can indirectly elicit neuronal depolarization by causing ATP depletion (Schousboe et al., $19896)$. The depletion of ATP and depolarization would then lead to the release of excitatory amino acids. This mechanism of cellular depolarization after mitochondrial injury could result from $\mathrm{NO}^{*}$ or, more probably, ONOO ${ }^{-}$and is consistent with our prior findings concerning an initial mitochondrial dysfunction in neuronal apoptosis and necrosis elicited by excitotoxins (Ankarcrona et al., 1995).

Because the overall concentration of glutamate in the culture medium was below the level that can elicit neuronal death in this system and did not increase during the course of the experiments, it is likely that if glutamate was involved, it was released locally near NMDA-R sites. This assumption is also supported by the observation that the effects observed in CGCs exposed to NO donors were independent of the total number of neurons, i.e., even when the number of neurons was reduced to $\sim 10 \%$, the relative extent of apoptosis and the sensitivity to NMDA-R antagonists were unchanged. Alternatively, the NO donors may have sensitized CGCs to the low amounts of NMDAlike agonists already present in the culture medium. The latter effect may have been the result of alterations of modulatory molecules, i.e., phosphorylating/dephosphorylating channel components. We are presently investigating these possibilities. 
Although dysregulated calcium homeostasis is recognized as a central event in cell death (Choi, 1985; Nicotera et al., 1985, 1992, 1994; Hahn et al., 1988; Lipton and Rosenberg, 1994; Dubinsky, 1995), its causative role has been disputed (Harman and Maxwell, 1995). Prevention of apoptosis in our experimental system by an intracellular calcium chelator (BAPTA) and by blocking calcium influx (MK-801, APV, or high $\mathrm{Mg}^{2+}$ levels) further strengthens the evidence that increased $\left[\mathrm{Ca}^{2+}\right]_{i}$ was not an epiphenomenon, but rather was directly involved in the apoptotic process.

Of the various species potentially generated by NO donors, ONOO ${ }^{-\prime}$ can nitrate and modify proteins (by oxidation to disulfide or by nitrotyrosine formation) (Beckman et al., 1994) or stimulate lipid peroxidation (Radi et al., 1991). ONOO seems to be formed on exposure of CGC cultures to SNOC or SNAP, as judged immunocytochemically by the finding of extensive tyrosine nitration in situ. The partial protection by SOD supports the involvement of $\mathrm{ONOO}^{-}$in CGC apoptosis. It is notable that the protective effect of the NMDA-R antagonists was apparently not due to a block in ONOO - formation. The observation that MK801 and APV failed to inhibit tyrosine nitration makes it unlikely that $\mathrm{Ca}^{2+}$ influx through NMDA-R channels was required to elicit the formation of $\mathrm{ONOO}^{-1}$ from $\mathrm{O}_{2}{ }^{--}$and NO donors. However, we do not exclude the possibility that $\mathrm{O}_{2}{ }^{\cdots}$ production following $\mathrm{Ca}^{2+}$ entry through NMDA-Rs may have contributed to the cytotoxic effects of the NO donors.

Neither glial cells exposed to the NO donors nor CGCs in the presence of NMDA-R antagonists under went apoptosis, despite tyrosine residues appearing heavily nitrated. These findings are consistent with the supposition that the apoptosis elicited by the NO donors in CGCs involves a specific event downstream to nitrotyrosine formation, which leads to subsequent NMDA-R stimulation. Alternatively, additional events different form tyrosine nitration, e.g., direct effects of other reactive species generated after exposure to the NO donors, may be required.

Cytoskeletal damage is a frequent component of neuronal cell death (Mattson, 1990; Lee et al., 1991). In particular, proteolytic disruption of cytoskeletal elements has been associated with the apoptotic program (Brancolini et al., 1995; Martin and Green, 1995; Kaylar et al., 1996). Modification and dissolution of F-actin are common features of both necrosis and apoptosis in several systems (Nicotera et al., 1992, 1994; Bonfoco et al., 1995a,b). In neuronal apoptosis, disruption of the microfilament network may be due to actin proteolysis mediated by ICE-like proteases (Brancolini et al., 1995; Kaylar et al., 1996). Our results suggest that disorganization of actin alone is not sufficient to produce the morphological changes associated with apoptosis in CGCs because cell shape and nuclear morphology apparently remained unaffected even when actin began to depolymerize. Nonetheless, when microtubules and lamins were also significantly disrupted, cells displayed marked morphological changes, including nuclear pyknosis followed by changes in membrane permeability to ethidium bromide (occurring between 12 and $18 \mathrm{~h}$ ). This is in agreement with our previous observations showing that primary microtubular damage can trigger CGC apoptosis (Bonfoco et al., 1995b).

In conclusion, we propose a novel mechanism to explain the neuronal demise elicited by NO donors such as SNOC or SNAP. This involves NO-mediated release of NMDA-R agonists with subsequent activation of NMDA-R channels and intracellular $\mathrm{Ca}^{2+}$ overload. The latter then activates downstream events leading to a sequential breakdown of major cytoskeletal proteins and apoptosis.

Acknowledgment: This study was supported by grants from the International Life Science Institute, the Swedish National Science Research Council (B-AA/BV $10173-$ 301), the Swedish Medical Rescarch Council (03X-2471), and the National Institutes of Health (RO EY05477, RO1 EY09024, and PO1 HD29587). We thank Dr. J. Eriksson for the gift of antibodies to lamins and Dr. J. Beckman for the antibodies against nitrotyrosines. We wish to thank Ms. Heike Neumann and Mr. Thomas Schmitz for excellent technical assistance.

\section{REFERENCES}

Ankarcrona M., Dypbukt 1. M., Bonfoco E., Zhivotovsky B., Orrenius S., Lipton S. A., Nicotera P. (1995) Glutamate-induced neuronal death: a succession of necrosis or apoptosis depending on mitochondrial function. Neuron 15, 961-973.

Beckman J. S., Ye Y. Z., Anderson P. G., Chen J., Accavitti M. A., Tarpey M. M., and White C. R. (1994) Extensive nitration of protein tyrosine in human atherosclerosis detected by immunohistochemistry. Biol. Chem. Hoppe Seyler 375, 81-88.

Bonfoco E., Krainc D., Ankarcrona M., Nicotera P., and Lipton S. A. (1995a) Apoptosis and necrosis: two distinci events induced respectively by mild and intense insults with NMDA or nitric oxide/superoxide in cottical cell cultures. Proc. Natl. Acad. Sci. USA 92, 72162-72166.

Bonfoco E., Ceccatelli S., Manzo L.., and Nicotera P. (1995b) Colchicine induces apoptosis in cerebellar granule cells. Exp. Cell Res. 218, $189-200$.

Brancolini C., Benedetti M., and Schneider C. (1995) Microfilament reorganization during apoptosis: the role of $\mathrm{G} \alpha \mathrm{s} 2$ a possible substrate for ICE-like protcases. EMBO J. 14, 5179-5190.

Bredt D. S. and Snyder S. H. (1989) Nitric oxide mediates glutamate linked enhancement of CGMP levels in the cerebellum. Proc. Nall. Acad. Sci. USA 86, 9030-9033.

Choi D. W. (1985) Glutamate neurotoxicity in cortical cell cultures is calcium dependent. Neurosci. Lett. 58, 293-297.

Davies J., Francis A. A., Jones A. W., and Watkins J. C. (1981) 2 Amino-5-phosphonovalerate (2APV), a potent and selective antagonist of amino acid-induced and synaptic excitation. Neurosci. Lett. 21, $77-8 \mathrm{~L}$.

Dawson V. I., Dawson T. M., London E. D., Bredt D. S., and Snyder S. H. (1991) Nitric oxide mediates glutamate neurotoxicity in primary cortical cultures. Proc. Natl. Acad. Sci. USA 88, 63686371.

Demerle-Pallardy C., Lonchampt M. O., Chabier P. E., and Braquet P. (1991) Absence of implication of the $/$-arginine/nitric oxide pathway on neuronal cell injury induced by $l$-glutamate or hypoxia. Biochem. Biophys. Res. Commun. 181, 456-464. 
Dubinsky J. M. (1995) Examination of the role of calcium in neuronal death. Ann. NY Acad. Sci. 679, 34-42.

Dugan L. L., Sensi S. L., Canzoniero L. M. T., Handran S. D., Rothman S. M., Lin T.-S., Goldberg M. P., and Choi D. W. (1995) Mitochondrial production of reactive oxygen species in cortical neurons following $N$-methyl-D-aspartate. $J$. Neurosci. $15,6377-6388$.

Garthwaite J. and Boulton C. L. (1995) Nitric oxide signalling in the central nervous system. Annu. Rev. Physiol. 57, 683-706.

Garthwaite J., Charles S. L., and Chess-Williams R. (1988) Endothelium-derived relaxing factor release on activation of NMDA receptors suggests role as intracellular messenger in the brain. Nature 336, 385-388.

Gross S. and Wolin M. S. (1995) Nitric oxide: pathophysiological mechanisms. Annu. Rev. Physiol. 57, 737-769.

Grynkiewicz G., Poenic M., and Tsien R. Y. (1985) A new generation of $\mathrm{Ca}^{2+}$ indicators with greatly improved fluorescence properties. J. Biol. Chem. 260, 3440-3450.

Hahn J. S., Aizenman E., and Lipton S. A. (1988) Central mammalian neurons normally resistant to glutamate toxicity are made sensitive by elevated extracellular $\mathrm{Ca}^{2+}$ : toxicity is blocked by the $N$-methy\}-D-aspartate antagonist MK-801. Proc. Natl. Acad. Sci. USA 85, 6556-6560.

Hanbauer I., Wink D., Osawa Y., Edelman G. M., and Gally J. A. (1992) Role of nitric oxide in NMDA-evoked release of ${ }^{3} \mathrm{H}-$ dopamine from striatal slices. Neuroreport 3, 409-412.

Hansson E. and Rönnbäck L. (1989) Primary cultures of astroglia and neurons from different brain regions, in A Dissection and Tissue Culture Manual of the Nervous System (Shahar A., De Vellis J., Vernadakis A., and Haber B., eds), pp. 92-104. Wiley-Liss, New York.

Harman A. W. and Maxwell M. J. (1995) An evaluation of the role of calcium in cell injury. Annu. Rev. Pharmacol. Toxicol. 35, $129-144$.

Kaylar C., Örd T., Testa M. P., Zhong L., and Bredesen D. E. (1996) Clcavage of actin by interleukin $1 \beta$ to teverse DNase I inhibition. Proc. Natl. Acad. Sci. USA 93, 2234-2238.

Lafon-Cazal M., Pietri S., Culcasi M., and Bockaert J. (1993) NMDA-dependent superoxide production and neurotoxicity. Nature 364, 535-537.

Lee K. S., Frank S., Vanderklish P., Arai A., and Lynch G. (1991) Inhibition of proteolysis protects hippocampal neurons from ischemia. Proc. Natl. Acad. Sci. USA 88, 7233-7237.

Lei S. Z., Pan Z. H., Aggarwal S. K., Chen H. S., Hartman J., Sucher N. J., and Lipton S. A. (1992) Effect of nitric oxide production on the redox modulatory site of the NMDA receptor channel complex. Neuron 8, 1087-1099.

Levi G., Aloisi F., Ciotti M. T., and Gallo V. (1984) Autoradiographic localization and depolarization-induced release of acidic amino acids in differentiating cerebellar granule cell cultures. Brain Res. 290, 77-86.

Lipton S. A. and Rosenberg P. A. (1994) Excitatory amino acids as a final common pathway for neurological disorders. $N$. Engl. J. Med. 330, 613-622.

Lipton S. A., Choi Y.-B., Pan Z.-H., Lei S. Z., Chen H.-S. V., Sucher N. J., Loscalzo J., Singel D. J., and Stamler J. S. (1993) A redox-based mechanism for the neuroprotective and neurode- structive effects of nitric oxide and related nitroso compounds. Nature 364, 626-632

Martin S. J. and Green D. R. (1995) Protease activation during apoptosis: death by a thousand cuts? Cell $\mathbf{8 2}, 349-352$.

Mattson M. (1990) Antigenic changes similar to those seen in neurofibrillary tangles are elicited by glutamate and $\mathrm{Ca}^{2+}$ influx in cultured hippocampal neurons. Neuron 2, 105-117.

Montague P. R., Gancayco C. D., Winn M. J., Marchase R. B., and Friedlander M. J. (1994) Role of NO production in NMDA receptor-mediated neurotransmitter release in cerebral cortex. Science 263, 973-977.

Nicotera P., Moore M., Mirabelli F., Bellomo G., and Orrenius S. (1985) Innibition of hepatocyte plasma membrane $\mathrm{Ca}^{2+}$. ATPase activity by menadione metabolism and its restoration by thiols. FEBS Lett. 181, 149-153.

Nicotera P., Bellomo G., and Orrenius S. (1992) Calcium-mediated mechanisms in chemically induced cell death. Annu. Rev. Pharmacol. Toxicol. 32, 449-470.

Nicotera P., Zhivotovsky B., Bellomo G., and Orrenius S. (1994) Ion signalling in apoptosis, in Apoptosis (Schimke R. T. and Mihich E., eds), pp. 97-115. Plenum Press, New York.

O'Dell T. J., Hawkins R. D., Kandel E. R., and Arancio O. (1991) Tests of the roles of two diffusible substances in long term potentiation: cvidence for nitric oxide as a possible early retrograde messenger. Proc. Natl. Acad. Sci. USA 88, 1285-1289.

Radi R., Beckman J. S., Bush K. M., and Freeman B. A. (1991) Peroxynitrite induced membrane lipid peroxidation. The cytotoxic potential of superoxide and nitric oxide. Arch. Biochem. Biophys. 228, $481-487$.

Richter C., Gogvadze V., Schlapbach R., Schweizer M., and Schlegel J. (1994) Nitric oxide kills hepatocytes by mobilizing mitochondrial calcium. Biochem. Biophys. Res. Commun. 205, $1143-1150$.

Schousboe A., Mejer E., Drejer J., and Hertz L. (1989a) Preparation of primary cultures of mouse (rat) cerebellar granule cells, in A Dissection and Tissue Culture Manual of the Nervous System (Shahar A., de Vellis J., Vernadakis A., and Haber B., eds), pp. 203-206. Alan R. Liss, New York.

Schousboe A., Frandsen A., and Drejer J. (1989b) Evvidence for evoked release of adenosine and glutamate from cultured cerebellar granule celis. Neurochem. Res. 14, 871-875.

Stamler J. S., Singel D. J., and Loscalzo J. (1992) Biochemistry of nitric oxide and its redox-activated forms. Science 258, 1898 1902.

Watkins J. C. and Evans R. H. (1981) Excitatory amino acid transmitters. Annu. Rev. Pharmacol. 21, 165-204.

Wong E. K., Kempt J. A., Priestley T., Knight A. R., Woodruff G. N, and Iversen L. L. (1986) The anticonvulsant MK+801 is a potent $N$-methyl-i)-aspartate antagonist. Proc. Natl. Acad. Sci. USA 83, 7104-7108.

Wyllie A. H. (1980) Glucocorticoid-induced thymocyte apoptosis is associated with endogenous endonuclease activation. Nature $284,555-556$.

Zhang J. and Snyder S. H. (1995) Nitric oxide in the nervous system. Annu. Rev. Pharmacol. Toxicol. 35, 213-233.

Zhang J., Dawson V. L., Dawson T. M., and Snyder S. (1994) Nitric oxide activation of poly-(ADP-ribose) synthetase in neurotoxicity. Science 263, 687-689. 